\title{
Potential Therapeutic Target Discovery by 2D-DIGE Proteomic Analysis in Mouse Models of Asthma
}

Florence Quesada Calvo, ${ }^{+, \neq}$Marianne Fillet, ${ }^{+, \neq}$Jenny Renaut, $^{\S}$ Céline Crahay, ${ }^{\dagger}$ Maud Gueders, ${ }^{\dagger}$ Jonathan Hacha, ${ }^{\dagger}$ Geneviève Paulissen, ${ }^{\dagger}$ Jean-Michel Foidart, ${ }^{\dagger}$ Agnes Noel, ${ }^{+}$Natacha Rocks, ${ }^{\dagger}$ Pierre Leprince, ${ }^{+, \neq}$and Didier Cataldo ${ }^{*,+, \neq}$

${ }^{+}$GIGA-research (GIGA-I ${ }^{3}$, GIGA-cancer, and GIGA-Neurosciences), University of Liege, Belgium

${ }^{\S}$ Department of Environment and Agrobiotechnologies, Center of Public Research-Gabriel Lippmann, Belvaux, Luxembourg

Supporting Information

ABSTRACT: As asthma physiopathology is complex and not fully understood to date; it is expected that new key mediators are still to be unveiled in this disease. The main objective of this study was to discover potential new target proteins with a molecular weight $>20 \mathrm{kDa}$ by using two-dimensional differential in-gel electrophoresis (2D-DIGE) on lung parenchyma extracts from control or allergen-exposed mice (ovalbumin). Two different mouse models leading to the development of acute airway inflammation ( 5 days allergen exposure) and airway remodeling (10 weeks allergen exposure) were used. This experimental setting allowed the discrimination of 33 protein spots in the acute inflammation model and 31 spots in the remodeling model displaying a differential expression. Several proteins were then identified by MALDI-TOF/

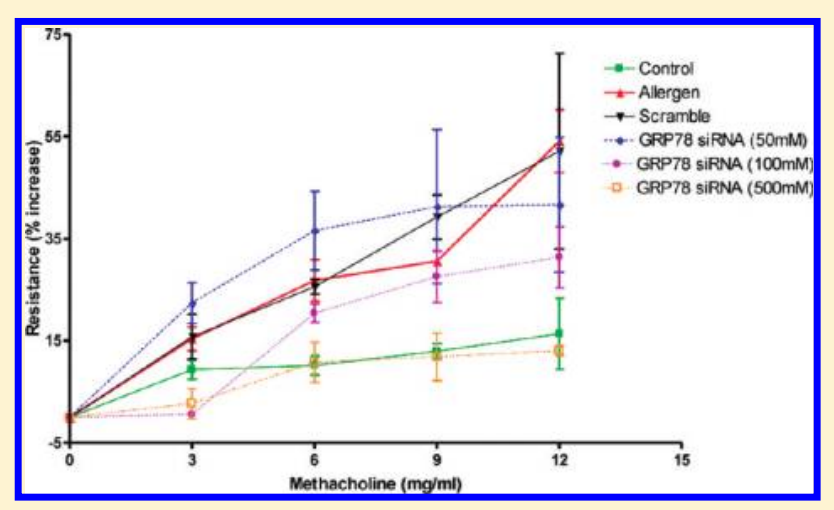
TOF MS. Among those differentially expressed proteins, PDIA6, GRP78, Annexin A6, hnRPA3, and Enolase display an increased expression in lung parenchyma from mice exposed to allergen for 5 days. Conversely, Apolipoprotein A1 was shown to be decreased after allergen exposure in the same model. Analysis on lung parenchyma of mice exposed to allergens for 10 weeks showed decreased calreticulin levels. Changes in the levels of those different mediators were confirmed by Western blot and immunohistochemical analysis. Interestingly, alveolar macrophages isolated from lungs in the acute inflammation model displayed enhanced levels of GRP78. Moreover, intratracheal instillation of anti-GRP78 siRNA in allergen-exposed animals led to a decrease in eosinophilic inflammation and bronchial hyperresponsiveness. This study unveils new mediators of potential importance that are up- and downregulated in asthma. Among up-regulated mediators, GRP-78 appears as a potential new therapeutic target worthy of further investigations.

KEYWORDS: proteomics, asthma, lung tissue profiling, MALDI-TOF/TOF, mouse model, inflammation, remodeling

\section{INTRODUCTION}

Asthma is a T-helper type 2-driven $\left(\mathrm{Th}_{2}\right)$ disease characterized by airway hyper-responsiveness and eosinophilic inflammation as well as histological changes of the bronchi including collagen deposition and mucus overproduction. ${ }^{1}$ Despite a growing knowledge of pathophysiological end points in asthma, comprehensive deciphering of the complex network of interacting mediators is still lacking.

To better understand the implication of new proteic mediators in intrinsic mechanisms of asthma, it is mandatory to know which genes, gene transcripts, protein species, and metabolites are differentially expressed during the course of the disease. ${ }^{1 \mathrm{~b}, \mathrm{c}}$ As proteins undergo many post-translational modifications that affect their structures and functions, the identification through appropriate proteomic approaches of their modified phenotypes and expression levels under pathological conditions may also convey useful information to discover new potential therapeutic targets. Proteomic studies of the lung parenchyma imply the purification and identification of individual proteins from a very complex mixture consisting of proteins expressed by resident and inflammatory cells as well as proteins from extracellular matrix and plasma. Protein separation by two-dimensional polyacrylamide gel electrophoresis (2D-electrophoresis) followed by identification through mass spectrometry are standard approaches in proteomics. In this study, we investigate the pathogenesis of asthma by a 2D-DIGE proteomic approach devoted to discriminate which proteins from mouse lungs are up- or down-regulated after acute or long-term allergen exposure.

\section{MATERIALS AND METHODS}

OVA-Induced Asthma Model

Specific pathogen-free Balb/c mice (6 weeks-old male) were used. Two different protocols consisting in acute or chronic

Received: May 26, 2011

Published: July 13, 2011 
exposure to allergen were applied and were approved by the Animal Ethics Committee of the University of Liège.

Mice were sensitized with 2 i.p. injections of $10 \mu \mathrm{g}$ of ovalbumine grade V (OVA) (Sigma, St. Louis, MO) adsorbed to aluminum hydroxide (Pierce Biotechnology Inc., Rockford, IL). Mice were then exposed to an aerosol of $1 \%$ OVA in phosphatebuffered saline (PBS) given by an ultrasonic nebulizer for $30 \mathrm{~min} /$ day during five consecutive days for short-term exposure (induction of acute inflammation, short-term exposure protocol (STE)) and during five days per even week until day 90 for long-term exposure (induction of a chronic remodeling, long-term exposure protocol (LTE)). Intratracheal instillations of target si-RNA (ON-TARGETplus SMARTpool siRNA, Dharmacon CO, IL) were performed the first, third, and last days of allergen exposure. Each experiment was performed with control mice $(n=10)$ and allergen-exposed mice $(n=10)$. Twenty-four hours after the last aerosol challenge, mice were anesthetized by intraperitoneal injection $(200 \mu \mathrm{L})$ of a mixture of ketamine $(10 \mathrm{mg} / \mathrm{mL}$, Merial, Brussels, Belgium) and xylazine $(1 \mathrm{mg} / \mathrm{mL}, \mathrm{VMD}$, Arendonk, Belgium). A tracheotomy was performed by insertion of a 20 gauge polyethylene catheter into the trachea. Mice were ventilated with a flexiVent small animal ventilator (SCIREQ, Montreal, Canada) to measure airway hyperresponsiveness to methacholine aerosol as described elsewhere. ${ }^{1 \mathrm{~b}}$

\section{Bronchoalveolar Lavage (BAL)}

After the sacrifice of mice, a bronchoalveolar lavage was performed using PBS/EDTA $0.05 \mathrm{mM}$ (Calbiochem, Darmstadt, Germany). After centrifugation of BAL, the supernatant was frozen at $-80^{\circ} \mathrm{C}$ for protein assessment and the cell pellet was resuspended in $1 \mathrm{~mL}$ PBS-EDTA $0.05 \mathrm{mM}$.

Total cells counts were performed by using a Coulter counter Z2 (Beckman, Brea, CA). Differential cell counts were performed after cytocentrifugation and staining with Diff-Quick (Dade, Belgium).

Primary alveolar macrophages were isolated from bronchoalveolar lavage fluid (BALF) by a simple adhesion method on standard plastic culture dishes.

\section{Pulmonary Histology}

The left lung was immediately frozen at $-80{ }^{\circ} \mathrm{C}$ until further processing and the right lung was infused with $4 \%$ paraformaldehyde, embedded in paraffin and processed for histology. Sections of $5 \mu \mathrm{m}$ thickness were cut off from paraffin blocks and were stained with hematoxylin-eosin. The peribronchial inflammation was estimated on hematoxylin-eosin-stained slides by a score calculated by quantification of peribronchial inflammatory cells (eosinophils, lymphocytes, macrophages, etc.), as previously described by Cataldo et al. ${ }^{2}$ After Congo Red staining, the eosinophilic infiltration in the airway walls was quantified by manual count in randomly selected bronchi and normalized to the perimeter of corresponding epithelial basement membrane defining an eosinophilic inflammatory score.

To assess airway mucus production, lung sections were analyzed using the Alcian Blue method. For each sample, positive cells percentage was evaluated. The peribronchial collagen deposition was estimated using Masson's Trichrome staining. Quantification was performed as previously described by Di Valentin et al. ${ }^{3}$ Blood was drawn from the heart at the time of sacrifice for serum OVA specific IgE measurement. ${ }^{4}$

\section{D-Difference Gel Electrophoresis (2D-DIGE)}

Lung Sample preparation and 2D-DIGE. All electrophoresis reagents and instrumentation were obtained from GE
Healthcare. Total proteins were extracted from lungs by an overnight incubation of crushed tissue with lysis buffer containing $7 \mathrm{M}$ Urea, $2 \mathrm{M}$ Thiourea, 2\% CHAPS and $50 \mathrm{mM}$ Tris $(\mathrm{pH} 9)$, followed by centrifugation at $13200 \mathrm{rpm}$ (20 min). After precipitation with the $2 \mathrm{D}$ Clean-Up Kit, protein concentrations were measured using the PlusONE 2-D Quant Kit. To label proteins, $25 \mu \mathrm{g}$ of each protein samples were incubated with 200 pmol of CyDye (Cy3, Cy5). A pool containing equal amounts of all samples was also prepared and labeled with Cy 2 to be used as an internal standard. The labeling was stopped by adding $10 \mathrm{mM}$ lysine. Experiments were performed in triplicate (3 independent sham- and 3 independent allergen-exposure samples in each model).

First-dimension isoelectric focusing was performed on 24-cm IPG strips, with a 3-10 linear $\mathrm{pH}$ gradient, in an IPGphor II instrument. The strips were rehydrated overnight with a mixture of two labeled samples ( $\mathrm{Cy} 3$ and $\mathrm{Cy} 5$ ) plus the internal standard in $450 \mu \mathrm{L}$ of a solution containing $7 \mathrm{M}$ urea, $2 \mathrm{M}$ thiourea, $2 \%$ ASB-14, 0.2\% dithiothreitol and 0.5\% IPG buffer 3-10 NL . Focusing was conducted for $1 \mathrm{~h}$ at $200 \mathrm{~V}, 1 \mathrm{~h}$ at $500 \mathrm{~V}, 1 \mathrm{~h}$ at 1000 $\mathrm{V}, 6 \mathrm{~h}$ at $8000 \mathrm{~V}$. The strips were then incubated with equilibration buffer (6 M urea, $50 \mathrm{mM}$ Tris $\mathrm{pH} 8.8,30 \%$ glycerol, $1.6 \%$ sodium dodecyl sulfate and a trace of bromophenol blue) containing $1 \%$ dithiothreitol and then $5 \%$ iodoacetamide, each time for $15 \mathrm{~min}$. They were then deposited on top of a $12.5 \%$ SDS-PAGE gel. The second-dimension separation was performed overnight in an Ettan Dalt II system.

\section{Gel Analysis}

Once the second dimension step was carried out, each gel ( 3 gels in each model) was scanned at three different wavelengths corresponding to the different CyDyes with a Typhoon 9400 Laser Scanner (GE Healthcare). Then all images were analyzed using DeCyder 2D Software 6.5 (GE Healthcare). A cutoff value was set at a 1.2-fold increase or decrease in normalized spot volume, and statistical differences in spot intensities were analyzed by Student $t$ test with $p<0.05$ (with visual inspection of the results).

\section{Protein Identification}

The spots of interest were excised from the gel with the Ettan Spot Picker robot (GE Healthcare) and were collected in 96-well plates designed for the digestion of proteins. Spots were washed and desalted in $50 \mathrm{mM}$ ammonium bicarbonate/methanol $(50 \% \mathrm{v} / \mathrm{v})$, followed by acetonitrile $(75 \% \mathrm{v} / \mathrm{v}, \mathrm{ACN})$. Then they were digested with Trypsin Gold (MS grade, Promega, Madison, WI, $10 \mathrm{mg} \mathrm{mL}^{-1}$ in $20 \mathrm{mM}$ ammonium bicarbonate) using the Ettan Digester robot (GE Healthcare) from the Ettan Spot Handling workstation. Automated spotting of the samples was carried out with the spotter of the same Workstation (GE Healthcare). Peptides dissolved in a 50\% ACN containing $0.5 \%$ TFA $(0.7 \mathrm{~mL})$ were spotted on MALDI-TOF disposable target plates (4800, Applied Biosystems, Foster City, CA) prior to the deposit of $0.7 \mathrm{~mL}$ of CHCA $(7 \mathrm{mg} / \mathrm{mL}, 50 \% \mathrm{v} / \mathrm{v} \mathrm{ACN}$, $0.1 \% \mathrm{v} / \mathrm{v}$ TFA, Sigma Aldrich, St. Louis, MO). Peptide mass determinations were carried out using the Applied Biosystems 4800 Proteomics Analyzer (Applied Biosystems). Both PMF and $\mathrm{MS} / \mathrm{MS}$ in reflectron mode analyses were carried out with the samples. Calibration was carried out with the peptide mass calibration kit for 4700 (Applied Biosystems). Proteins were identified by searching in Swiss-Prot, TREMBL and NCBI databases using MASCOT (Matrix Science, www.matrixscience.com, London, U.K.). All searches were carried out using a mass window 


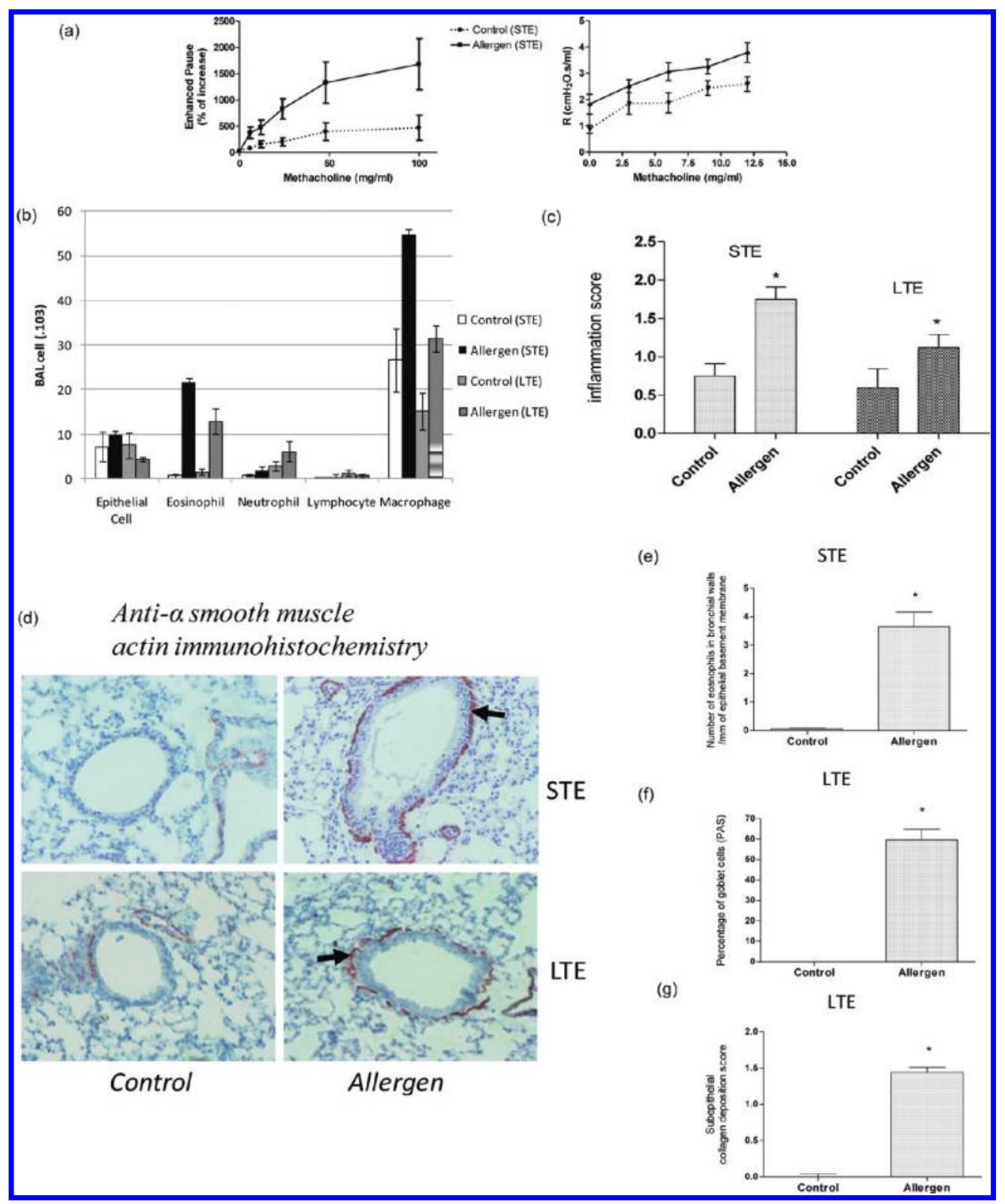

Figure 1. Characterization of airway responsiveness, inflammation and remodeling. (a) Measurement of airway responsiveness: Airway resistances (Enhanced pause $-R$ ) were measured after increasing concentrations of methacholine $(0-100 \mathrm{mg} / \mathrm{mL}-0-12 \mathrm{mg} / \mathrm{mL}$ ). Measurements were performed by barometric plethysmography (left panel) or by the forced oscillation technique (FlexiVent, right panel) $24 \mathrm{~h}$ after the last allergen or PBS exposure. (b) Differential BAL fluid cell counts were performed after cytocentrifugation in both acute inflammation, short-term exposure (STE), and chronic remodeling protocols, long-term exposure (LTE). (c) Inflammation score measured on H\&E stained $5 \mu$ m sections (see Materials and Methods for description of inflammation score). (d) Characterization of allergen-induced remodeling: Morphometric quantification of smooth muscle alphaactin in the airway walls of mice after short- (acute inflammation protocol) or long-term (chronic remodeling protocol) exposure to allergens. (e) Tissue eosinophil infiltration was assessed in the peribronchial area by congo red staining and cell numbers were counted by microscope examination. ( $\mathrm{f}$ and $\mathrm{g}$ ) Morphometric quantification of goblet cells and subepithelial collagen deposition in the airways of mice after chronic exposure to allergen after PAS and Masson's trichrome staining, respectively.

of 100 ppm and with "Mus musculus" as taxonomy in NCBInr database (http://www.ncbi.nlm.nih.gov/) and 0.5 Da of tolerance on MS/MS fragments. The search parameters allowed fixed modifications for cysteine (carboxyamidomethylation) and methionine (oxidation) and variable modifications on tryptophan (double oxidation or kynurenin). Two missed cleavages were allowed, and the peptide charge was set at +1 . All of the identifications were manually verified and validated.

\section{Protein Validation Approaches}

Western Blot. Lung sample proteins were separated under reducing conditions using NuPage $12 \%$ Bis-Tris Gels (Invitrogen, Carlsbad, CA), transferred, and incubated with primary antibodies: rabbit anti-hnRNP A3 (Santa Cruz Biotechnology, Foster City, CA), chicken anti-PDIA6 (Abcam, U.K.), rabbit anti-Calreticulin (Santa Cruz Biotechnology), rabbit antiAnnexin VI (Abcam), rabbit anti-Apolipoprotein Al (Abcam), rabbit anti-GRP78 $\mathrm{BiP}$ (Abcam). 


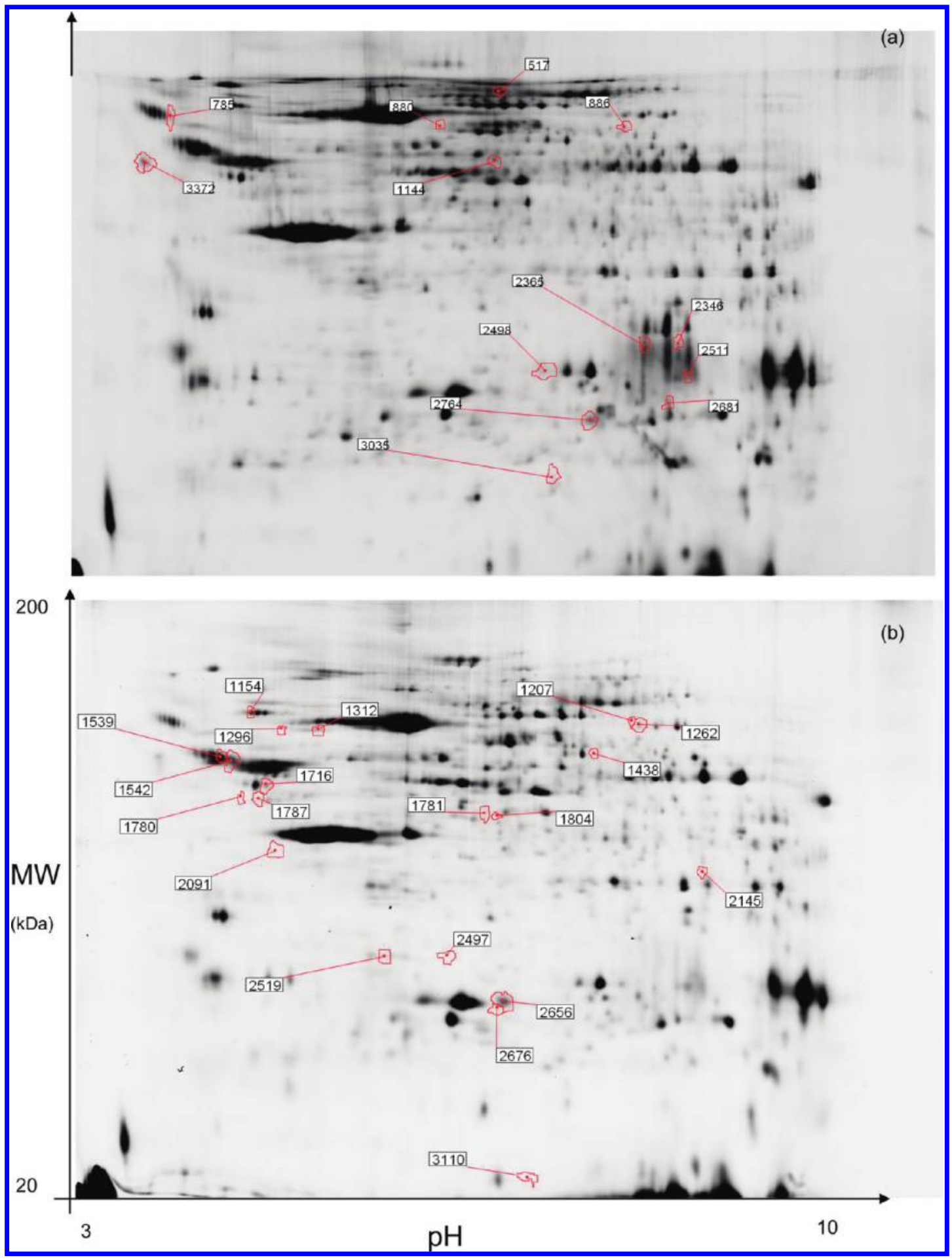

Figure 2. Representative 2D-DIGE: Distribution of differentially expressed protein spots mice in lung extract after short-term exposure (a) and in longterm exposure (b) to allergens or placebo (PBS). Each spot name relates to data shown in Table 1. Samples from one control and one allergen-exposed mice and internal standard were loaded in each gel (3 different gels in each model). Internal standard was a pool of all samples from acute and chronic model.

Incubation with corresponding secondary antibody conjugated to horseradish peroxidase was followed and signal was detected using the enhanced chemiluminescence (ECL) kit (Perkin-Elmer, Waltham, MA) as recommended by the manufacturer.

Western blots were analyzed by densitometry scanning. The intensity of each band was measured with the Quantity One software (Biorad, Hercules, CA). Proteins levels were normalized using GAPDH signal used as an internal standard.

\section{Immunohistochemistry}

Endogenous peroxidase activity was blocked by incubating tissue sections in $3 \%$ hydrogen peroxide $\left(\mathrm{H}_{2} \mathrm{O}_{2}\right)$. After deparaffinization, 
Table 1.

\begin{tabular}{|c|c|c|c|c|c|c|c|c|c|c|}
\hline & Master & Protein Symbol & Swiss prot & Mass & pl & Score & No of peptide & Coverage (\%) & pValue & Fold \\
\hline \multirow[t]{24}{*}{ STE } & 1438 EH-domain containing 1 & Ehd1 & Q80zzo & 60862 & 6,35 & 221 & 30 & 84,65 & 0,029 & $-1,32$ \\
\hline & 1539 prolyl 4-hydroxylase, beta polypeptide & P4HB & P09103 & 57502 & 4,79 & 158 & 18 & 54,03 & 0,044 & $-1,71$ \\
\hline & 1542 tubulin, beta 5 & Tubb5 & P99024 & 50367 & 4,78 & 252 & 28 & 100 & 0,044 & $-1,57$ \\
\hline & 1716 unnamed protein product & / & I & 54503 & I & 33 & 6 & / & 0,048 & $-1,41$ \\
\hline & 2497 Chloride intracellular channel 5 & CuCs & Q8BXK9 & 28488 & 5,64 & 219 & 27 & 100 & 0,04 & $-1,26$ \\
\hline & 2519 purine-nucleoside phosphorylase & $\mathrm{Np}$ & P23492 & 32748 & 5,78 & 68 & 13 & 57,71 & 0,011 & $-1,36$ \\
\hline & 2656 indolethylamine $\mathrm{N}$-methyltransferase & INMT & P40936 & 30116 & 6 & 304 & 21 & 81,06 & 0,043 & $-1,88$ \\
\hline & 2676 indolethylamine $\mathrm{N}$-methyltransferase & INMT & P40936 & 30116 & 6 & 176 & 18 & 78,41 & 0,029 & $-1,53$ \\
\hline & 2812 apolipoprotein A-I precursor & APOA1 & Q00623 & 60454 & 5,64 & 118 & 22 & 98,1 & 0,04 & $-1,26$ \\
\hline & 3110 peptidylprolyl isomerase A & PPWD1 & Q8CEC6 & 18211 & 6,58 & 302 & 15 & 19,19 & 0,042 & $-1,67$ \\
\hline & 116278 kDa glucose-regulated protein precursor (GRP78) & HSPA5 & P20029 & 72667 & 5,07 & 318 & 39 & 80,76 & 0,024 & 1,64 \\
\hline & 1207 Far upstream element-binding protein 1 (FUSE-binding protein1) & FUBP1 & Q91Wנ8 & 68908 & 7,73 & 94 & 18 & 31,64 & 0,0025 & 1,33 \\
\hline & 1262 5'-nucleotidase, cytosolic II & Nt5c2 & Q3V1L4 & 65491 & 5,81 & 35 & 5 & 11,96 & 0,021 & 1,4 \\
\hline & 1296 lymphocyte cytosolic protein 1 & LCP1 & Q61233 & 71004 & 5,2 & 170 & 30 & 57,25 & 0,03 & 1,5 \\
\hline & 1312 Annexin A6 & ANXA6 & P14824 & 76646 & 5,34 & 125 & 28 & 52,89 & 0,0007 & 1,36 \\
\hline & 1780 Protein disulfide isomerase associated 6 & PDIA6 & Q922R8 & 49122 & 5 & 116 & 19 & 58,18 & 0,0038 & 1,45 \\
\hline & 1781 hypothetical protein LOC433182 (Enolase) & Eno1 & Q5FW97 & 47597 & 6,37 & 116 & 22 & 78,34 & 0,039 & 1,54 \\
\hline & 1787 Protein disulfide isomerase associated 6 & PDIAG & Q922R8 & 49122 & 5 & 326 & 13 & 29,31 & 0,036 & 1,51 \\
\hline & 1804 protein phosphatase- 1 regulatory subunit 7 & Ppp1r7 & Q3UM45 & 41524 & 4,85 & 42 & 5 & 16,89 & 0,023 & 3,07 \\
\hline & 2054 parathyroid hormone-related peptide & Pthlh & P22858 & 20154 & 10,74 & 37 & 3 & 27,42 & 0,023 & 3,07 \\
\hline & 2091 gamma actin & ACTG1 & P63260 & 41591 & 5,31 & 224 & 13 & 42,13 & 0,032 & 1,39 \\
\hline & 2145 thiosulfate sulfurtransferase & Tst & P52196 & 33769 & 7,71 & 93 & 9 & 31,31 & 0,04 & 1,27 \\
\hline & 2145 Hnrpa3 protein & HNRNPA3 & Q8BG05 & 34696 & 9,1 & 70 & 4 & 15,04 & 0,04 & 1,27 \\
\hline & 2179 putative beta-actin (aa $27-375$ ) & Actb & P60710 & 41737 & 5,29 & 436 & 16 & 56,89 & 0,019 & $-2,21$ \\
\hline \multirow[t]{14}{*}{ LTE } & 785 serine (or cysteine)proteinase inhibitor, clade A, member $3 \mathrm{~K}$ & Serpina3k & P07759 & 47261 & 5,05 & 284 & 14 & 37,08 & 0,019 & $-2,21$ \\
\hline & 886 very-long-chain acyl-COA dehydrogenase & Acadvl & P50544 & 62468 & 8,91 & 81 & 10 & 20,58 & 0,044 & $-1,31$ \\
\hline & 1144 tubulin, alpha6 & Tuba1c & P68373 & 50722 & 4,96 & 119 & 9 & 27,39 & 0,022 & $-2,15$ \\
\hline & 3044 Vacuolar protein sorting 29 & VSP29 & Q9QZ88 & 20707 & 6,29 & 77 & 10 & 46,15 & 0,027 & $-1,51$ \\
\hline & 3381 calreticulin & CALR & P14211 & 48216 & 4,33 & 54 & 7 & 13,46 & 0,022 & $-1,44$ \\
\hline & 517 transferrin & Tf & Q92111 & 79000 & 6,94 & 126 & 12 & 17,36 & 0,013 & 1,44 \\
\hline & 880 dihydropyrimidinase-like 2 & Dpys12 & 008553 & 62830 & 5,95 & 71 & 11 & 19,23 & 0,0087 & 2,12 \\
\hline & 880 Protein disulfide isomerase $\mathrm{A} 3$ & PDIA3 & P27773 & 57099 & 5,88 & 236 & 24 & 45 & 0,0087 & 2,12 \\
\hline & 2346 alpha-globin & Hba-a1 & Q61287 & 6244 & 7,97 & 103 & 5 & 30,98 & 0,016 & 1,7 \\
\hline & 2365 alpha-globin & Hba-a1 & Q61287 & 6244 & 7,97 & 120 & 5 & 32,39 & 0,027 & 1,93 \\
\hline & 2499 Carbonic anhydrase 2 & CA2 & P00920 & 29186 & 6,49 & 76 & 9 & 37 & 0,0046 & 1,68 \\
\hline & 2503 Carbonic anhydrase 2 & CA2 & P00920 & 29186 & 6,49 & 272 & 19 & 91,15 & 0,024 & 2,28 \\
\hline & 2681 Glutathione S-transferase Mu 2 & GSTM2 & P15626 & 25871 & 6,91 & 129 & 16 & 60 & 0,022 & 1,2 \\
\hline & 2773 gluthatione S-transferase, mu 2 & Gstm2 & P15626 & 26015 & 6,91 & 80 & 13 & 38,99 & 0,023 & 1,7 \\
\hline
\end{tabular}

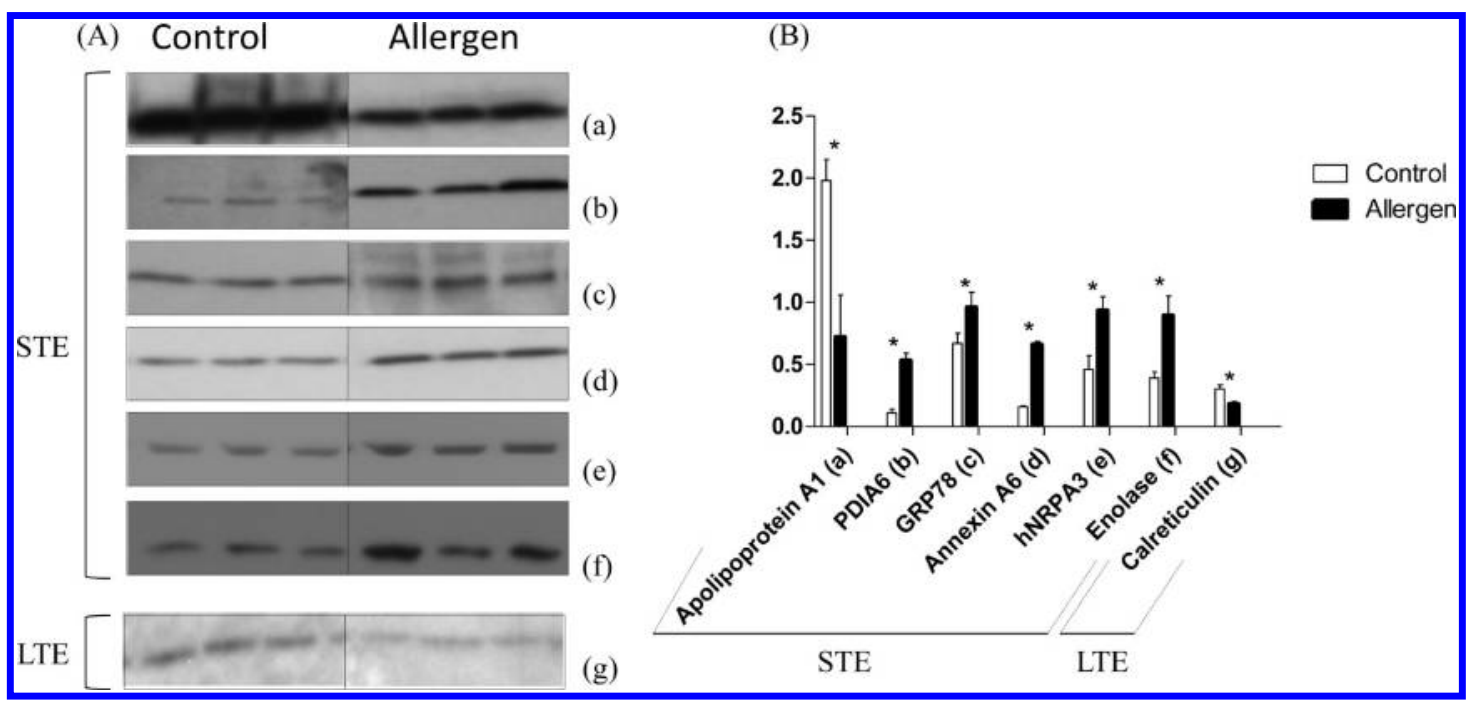

Figure 3. Validation of identified protein candidates. Western blot analyses for (a) Apolipoprotein A1, (b) PDIA6, (c) GRP78, (d) Annexin A6, (e) hNRPA3, (f) Enolase of representative lung extracts from acute inflammatory protocol and (g) calreticulin from chronic remodeling protocol. Only 3 representative examples of each group in both models are showed in this figure, but statistical analyses were performed on all studied samples. $\left({ }^{*}\right.$ Mann-Whitney U test, $p$-value $\left.<0.05\right)$.

slides were permeabilized in 1\% triton X-100/PBS and nonspecific binding was blocked in normal goat serum (Annexin A6, Enolase and Calreticulin) or in 10\% BSA (PDIA6, GRP78, hnRNPA3 and aSMA). Tissues sections were then incubated with rabbit antiAnnexin VI (Abcam), rabbit anti-Enolase (Abcam), rabbit antiCalreticulin (Santa Cruz Biotechnology), rabbit anti-PDIA6
(Abcam), rabbit anti-GRP78 (Abcam), rabbit anti-hnRNPA3 (Santa Cruz Biotechnology) or mouse anti- $\alpha$ smooth muscle actin (Dako). Secondary antibodies were coupled either to FITC-POD ( $\alpha$ SMA) or to biotin (Annexin A6, Enolase, Calreticulin, $\alpha$ SMA, PDIA6, GRP78 and hnRNPA3). Peroxidase activity was revealed using 3-amino, 9-ethyl-carbazole (AEC) staining ( $\alpha$ SMA) or 
3-3'diaminobenzidine hydrochlorid kit (DAB) after incubation with streptavidin/HRP complex (Dako).

\section{Cell Isolation and Flow Cytometry}

Lungs were digested one hour at $37^{\circ} \mathrm{C}$ in $1 \mathrm{mg} / \mathrm{mL}$ collagenase A (Roche, Vilvoorde, Belgium) in HBSS. Interstitial macrophages (IMs) and alveolar macrophages (AMs) were sorted by flow cytometry (FACSAria, Becton Dickinson) based on their differential F4-80/CD11c expression (eBioscience, San Diego, CA and BD Biosciences, Franklin Lakes, NJ, respectively). Staining reactions were performed at $4{ }^{\circ} \mathrm{C}$. Cells were incubated with $2.4 \mathrm{G} 2 \mathrm{Fc}$ receptor (CD16/CD32) antibodies to reduce nonspecific binding.

\section{Statistical Analysis}

Comparison (Western blot and immunohistochemistry) between groups ( $n=10$ /group) were performed using MannWhitney test. Results are reported as mean \pm SEM. $P$-values $<0.05$ were considered as statistically significant.

\section{RESULTS}

Characterization of Allergen-Induced Airway Responsiveness, Inflammation and Remodeling

To assess the validity of asthma models used in 2D-DIGE study, the airway responsiveness was measured $24 \mathrm{~h}$ after the last allergen challenge by direct measurement of lung resistances by using the Flexivent ventilator and whole body plethysmography (enhanced pause (Penh) measurement) and found to be increased after allergen exposure in short-term exposure model (Figure 1a). As expected from previous studies, ${ }^{5}$ no difference was seen in the long term exposure model (data not shown).

Eosinophil numbers were significantly increased in BAL and in bronchial walls after allergen exposure (Figure $1 \mathrm{~b}-\mathrm{e}$ ). Allergenexposed mice displayed significantly more inflammatory cells in the peribronchial area on lung sections stained by $H \& E$ (Figure 1c). Representative picture of this results is presented in the Supporting Information (Figure 1SA). Features of airway remodeling were studied on histologic lung sections by using specific stainings. Alpha-smooth muscle actin staining showed that mice exposed to allergens for short and long durations (STE and LTE protocols) displayed thickened layers of smooth muscle cells in the airway walls (Figure 1d). Numbers of PAS-positive mucous-secreting Goblet cells were higher after chronic allergen exposure (Figure 1f). Collagen deposition assessed by Masson's trichrome staining was increased only after long-term exposure (LTE) to allergens (Figure 1g). Representative picture of this result is presented in the Supporting Information (Figure 1SB). Finally, anti-OVA specific IgE levels were found to be significantly elevated in allergen immunized and challenged mice as compared to allergen immunized and sham (PBS) exposed animals (data not shown).

\section{Proteomic Profile after 2D-DIGE Analysis}

2D-DIGE was used to identify proteins that are differentially expressed in allergen-exposed mice in acute inflammation and chronic remodeling models. Spots general distribution patterns were similar in both groups of each model (Figure $2 a-b$ ). Nevertheless, 64 different spots showed a greater than 1.2-fold change in normalized volume with a $t$-test score below 0.05 in the Biological Variation Analysis in allergen-exposed versus sham-exposed animals, therefore suggesting differential protein expression. In the short term exposure protocol, the intensity of

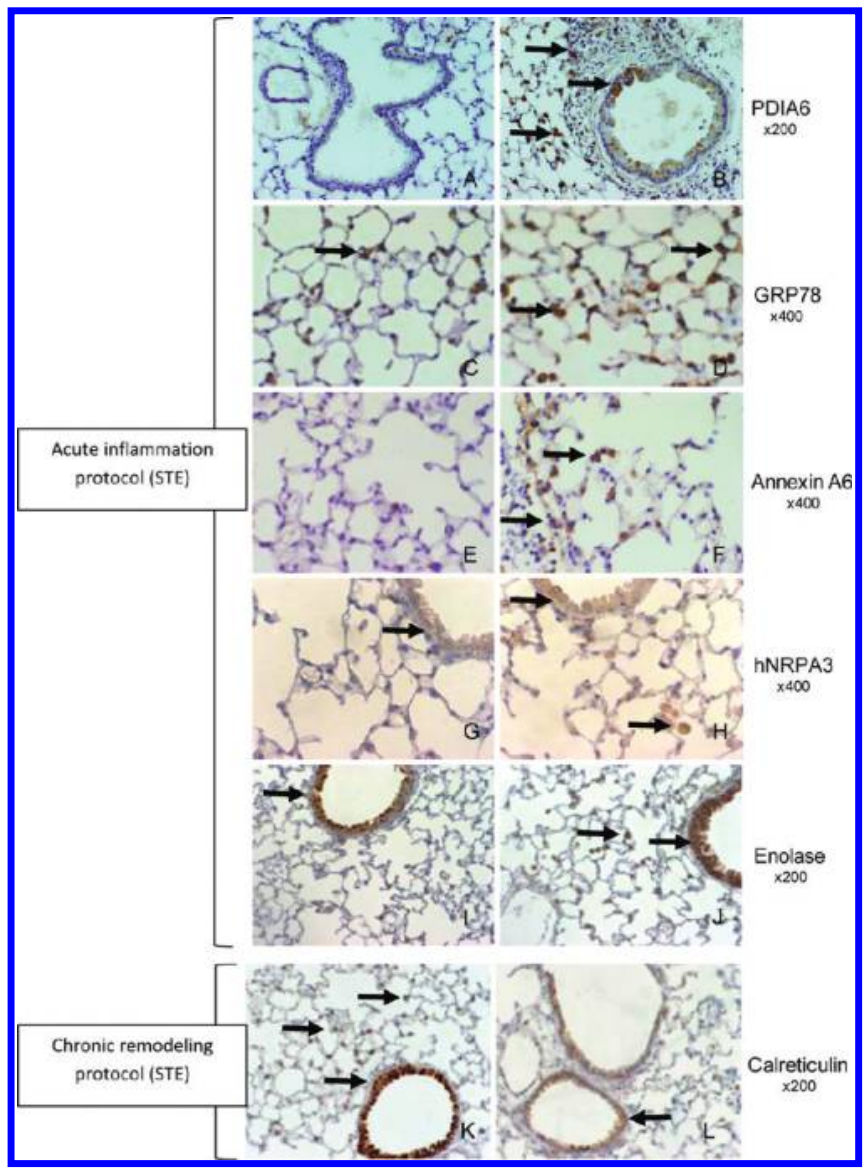

Figure 4. Validation of identified protein candidates by immunohistochemistry. (A-J) Immunohistochemical analysis of sham or allergenexposed mice lung sections using antibodies directed against $(\mathrm{A}-\mathrm{B})$ PDIA6, (C-D) GRP78, (E-F) Annexin A6, (G-H) hNRPA3, and (I-J) Enolase from short-term exposed mice (STE). (K-L) Immunohistochemical analysis of sham or allergen-exposed mice lung sections using antibody directed against Calreticulin from long-term exposed mice (LTE).

19 spots increased while the intensity of 14 spots decreased upon allergen exposure. In contrast, when mice were exposed for longer periods to allergens, only 9 spots were increased while 22 spots were decreased after allergen exposure. The list of proteins identified by MALDI-TOF/TOF after spot picking is shown in Table 1 and most are illustrated in Figure 2. Nine of the spots failed to yield any identification, giving an overall identification success rate of $70 \%$ for this study.

\section{Validation of 2D-DIGE Results by Western Blot Analysis}

To confirm the 2D-DIGE results and protein identifications by MALDI-TOF/TOF, we performed Western blots using antibodies against 7 different proteins selected in Table 1, based on antibody availability. We therefore confirmed that PDIA6, GRP78, Annexin A6, hnRNPA3, and Enolase production was increased in lung parenchyma after short-term allergen exposure while Apolipoprotein Al was less abundant in the same conditions only in the short-term exposure model (Figure 3). Similar results were obtained after long-term exposure (data not shown). Calreticulin levels were less important in lung parenchyma after long allergen exposure protocols as described in Figure 3, again increased level of this protein were demonstrated in the shortterm exposure model (data not shown). 


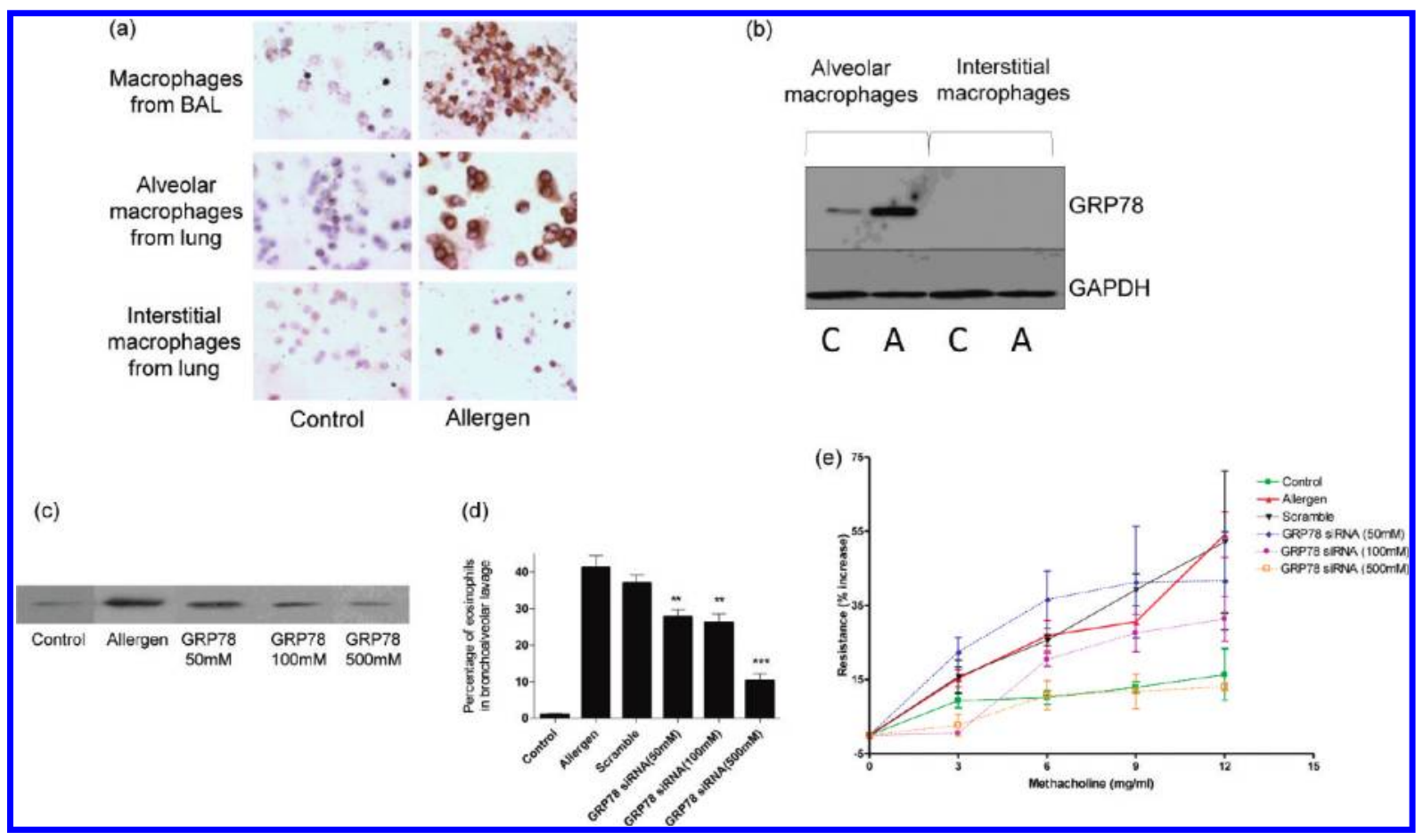

Figure 5. Modulation of GRP78 production in macrophages and GRP78 inhibition experiments. (a) Immunohistochemistry performed with an antiGRP78 antibody on cultured bronchoalveolar lavage (BAL) macrophages and interstitial or alveolar macrophages isolated from whole lung extracts of sham or allergen-exposed mice (acute inflammation protocol (STE)). (b) Western blot with anti-GRP78 antibody performed on interstitial and alveolar macrophages isolated from lungs of sham or allergen-exposed mice (acute inflammation protocol (STE)). These macrophages were sorted by flow cytometry (see Materials and Methods section). (c) Levels of GRP78, measured by Western blot, were decreased after instillations of siRNA (50, 100, and $500 \mathrm{mM}$ ). (d) GRP78 inhibition experiments: Anti GRP78 siRNA (50, 100, and $500 \mathrm{mM}$ ) was administered intratrachelly in mice and eosinophil percentages in BAL fluid were measured. (e) Measurement of airway resistance.

\section{Validation of 2D-DIGE Results by Immunohistochemistry}

After short time exposure to allergens (STE), PDIA6 immunostaining was more frequently detectable in inflamed lungs as compared to controls (Figure $4 a-b$ ). In particular, an intense staining was observed in alveolar macrophages and some bronchial epithelial cells. GRP78, Annexin A6, hnRNPA3, and Enolase proteins (Figure $4 c-h$ ) were also detectable by immunohistochemistry with far higher intensities in the allergen-exposed mice. For those proteins, staining was mainly located in lung parenchyma macrophages and in bronchial epithelial cells of allergen-exposed mice.

\section{Study of GRP78 Expression in Alveolar and Interstitial Macrophages}

An immunocytochemical analysis of GRP78 production was performed on cells (macrophages) cultured from bronchoalveolar lavage and on macrophages from lung parenchyma sorted by flow cytometry and cytocentrifuged. In BAL cells, anti-GRP78 stained a majority of cells from allergen-exposed as compared to shamexposed mice (Figure 5a). Sorted alveolar macrophages (AM) and interstitial macrophages (IM) were cytocentrifuged in order to perform an immunocytological staining that labeled mostly AM as compared to IM population from allergen-exposed mice. GRP78 protein levels measured by Western blot were also largely increased in AM from allergen-exposed as compared to sham-exposed mice or to IM in both conditions (Figure $5 \mathrm{~b}$ ).

\section{Validation of GRP78 as a Potential Therapeutic Target in Asthma}

To demonstrate a possible pathological role related to higher GRP78 levels observed in lungs of mice after allergen exposure, we tested the effects of reducing the expression of GRP78 in vivo using anti-GRP78 siRNA treatment. GRP78 levels measured by Western blot in protein extracts from lung parenchyma were decreased in a dose-dependent manner following intratracheal instillation of siRNA (Figure 5c). GRP78 inhibition by intratracheal instillation of siRNA led to a significant dose-dependent decrease of eosinophil percentages in BAL and bronchial hyperresponsiveness from allergen-exposed mice (Figure $5 \mathrm{~d}-\mathrm{e}$ ).

\section{DISCUSSION}

There is a considerable interest in proteomic studies performed on samples from patients or animals suffering from chronic inflammatory diseases such as asthma. These techniques allowing a large-scale study of proteins unveil new pathophysiological mechanisms and identify new biomarkers and/or potential therapeutic targets. We previously reported a proteomic study in a preclinical asthma model using SELDI-TOF-MS for the detection of low molecular weight (mostly $<20 \mathrm{kDa}$ ) proteins. ${ }^{6}$ The present study has been designed to address the modulation of high molecular weight proteins in the same animal models of asthma and has been performed by using 2D-DIGE since this technique is recognized as being able to resolve complex biological samples. ${ }^{7}$

In the present study, mice were characterized regarding main features of asthma and displayed a full asthmatic phenotype including bronchial hyperresponsiveness, eosinophilic inflammation, and bronchial remodeling. 2D-DIGE analysis performed on whole lung extracts of individual animals revealed, among other differentially expressed proteins, increased amounts of PDIA6, 
GRP78, Annexin A6, hnRPA3, and Enolase in lung parenchyma from mice exposed to allergens for 5 days. On the contrary, Apolipoprotein A1 was found to decrease in the same model after allergen exposure. Moreover, Calreticulin was shown to decrease in lung parenchyma of mice exposed to allergens for 10 weeks. To the best of our knowledge, this study reports for the first time a 2D-DIGE study of proteins differentially expressed in longterm allergen-exposed animals allowing the study of key events occurring when airway remodeling establishes.

The rationale to perform the present study on two mice models of asthma is that these models allow a full characterization of the asthmatic phenotype since they have been previously shown to involve a reproducible inflammation and enable the study of lung parenchyma after animal sacrifice. ${ }^{4}$ In particular, we used the $\mathrm{Th}_{2}$-prone BALB/c mice known to constitute a reliable model to study asthma phenotype. ${ }^{4,5,8} \mathrm{We}$ validated our findings regarding protein differential expression in our 2D-DIGE analysis by Western blotting and immunohistochemical analysis in order to generate additional information about the cells responsible for an overexpression of various lung proteins in the context of allergen-induced inflammation. Although previous authors have studied asthma models by using the 2D-DIGE technique, the present study is the first to compare both short-term and long-term allergen exposures regarding protein differential expression. ${ }^{9}$ Some of the previous authors have also studied nonallergic asthma, including some models of occupational asthma induced by various agents including toluene diisocyanate. ${ }^{10}$ Although almost all previous 2D-DIGE studies have been performed either on BALF or subcompartments of lung tissue (e.g., lymph nodes or bronchial epithelium), we decided to conduct the present on whole lung homogenized to unveil potential new informations on the processes leading to airway inflammation and remodeling without focusing on the immunological mechanisms. ${ }^{9 a, b, 10 a, 11}$ Some authors have also used 2D-DIGE studies in such models to study the effects of steroids on allergen-induced inflammation. ${ }^{12}$ Very recently, Verrils et al. reported that 2D-DIGE applied to human serum might help to diagnose asthma and COPD by defining a set of upregulated proteins. ${ }^{13}$

Some of the proteins like Enolase, Apolipoprotein A1, Calreticulin, and GRP78 identified in our study are in the "TOP 15 most often identified differentially expressed proteins" established by Petrak et al. ${ }^{14}$ This reinforces the probability that those mediators could be considers key players in various biological pathways.

Among validated proteins, GRP78, PDIA6, and calreticulin are considered chaperone proteins. These proteins are mainly found to be associated in the EIF2 complex and are often implicated in cellular response to stress. Therefore, it is potentially interesting to study these proteins since increased cellular stress occurs during the development of allergic inflammation in the airway and could account per se for the increased levels of these proteins detected after acute inflammation protocol.

We report that the production of GRP78 is higher in the lung parenchyma in the context of acute allergen-induced inflammation and is detectable in alveolar macrophages in immunohistochemistry. To document the implication of GRP78 in pathophysiological mechanisms of asthma, we performed a RNA interference study by instillation of anti-GRP78 siRNA, allowing a significant decrease of GRP78 protein expression in the lungs. Once GRP78 production was inhibited, bronchial responsiveness and airway inflammation decreased, suggesting that inhibition of GRP78, a target unveiled by this proteomic study, might become a potential new therapeutic tool in asthma. This is the first time that a single study reports mediator found to be overexpressed in asthma models that effectively play a determinant role in the pathophysiology of allergen-induced airway inflammation. GRP78 expression has been previously reported to increase in chronic diseases such as atherosclerosis, ${ }_{15}$ neurologic disorders (Alzheimer's and Parkinson's disease), ${ }^{16}$ and cancer. ${ }^{17}$ This protein belongs to the chaperone protein family but probably plays multiple roles in vivo since it is detected both in the endoplasmic reticulum and at the plasma membrane where it interacts with various extracellular signals such as $\alpha 2$ macroglobulin, Plasminogen Kringle 5, T-Cadherin, etc. ${ }^{18}$ To date, the precise mechanisms implicating GRP78 in the cascade leading to the asthma phenotype is not fully deciphered, but one can hypothesize that stress-induced damages following allergen exposure (activated cells releasing proteases or oxidants) might be responsible for a GRP78-dependent activation of various intracellular pathways. Indeed, activation of Phosphatidylinositol 3-kinases/Akt and Ras/MAPkinase by a GRP78 dependent mechanism has been reported in cancer and inflammation, therefore leading to the activation of $\mathrm{NF} \kappa \mathrm{B}$ and $\mathrm{mdm} 2$ among other pathways. ${ }^{18 c, 19}$ Taking into account the properties of these intracellular pathways and their implication in cell survival and proliferation, one can expect that they could also play a role in airway remodeling reported in asthma including smooth muscle cells proliferation and modified epithelium and conjunctive tissue cells such as fibroblasts. ${ }^{20}$ As GRP78 has been recognized as an angiogenesis promoting agent, a role for this protein in chronic asthma-related angiogenesis could also be hypothesized. ${ }^{21}$ Inflammatory cell survival could also be affected by GRP78 stimulation. ${ }^{22}$ Interestingly, it was recently reported that epigallocatechins gallate, displaying biological activities as GRP78 inhibitor, is effective in controlling allergen-induced asthma in a Guinea Pigs model of asthma confirming that our finding of a key role for GRP78 in asthma physiopathology should be studied more intensively. ${ }^{23}$ Further studies using GRP78 deletion mouse transgenes could be informative, but they would require a targeted deletion in adult lung tissue since the GRP78-deficient mice die early after birth. ${ }^{24}$

PDIA6 is another protein up-regulated in our model of acute asthma. This protein also acts as a folding chaperone and is mainly located in the endoplasmic reticulum but also on the cell surface. $^{25}$ Interestingly, it has been previously reported that PDIA6 gene silencing interferes with the shedding of the major histocompatibility complex class-I-related ligand (MICA), which in turn has been linked to asthma when genetic polymorphisms are sought in large human populations. ${ }^{26}$

Annexin A6 plays a role in calcium handling in muscle cells. ${ }^{27}$ This protein could therefore potentially interact with muscle function and might take part to smooth muscle hyperresponsiveness found in asthma. ${ }^{27}$ The phospholipidbinding protein Annexin A6 is a potential S100A8/A9 binding protein. ${ }^{28}$ Halayko et al. hypothesized that S100A8/A9 could be a mediator in asthma inflammation and remodeling since S100A8/A9 induce cell proliferation, inflammation, collagen synthesis, and cell migration. ${ }^{29}$

HNRPA3 was reported as overexpressed in acute asthma in our model. This protein is probably active in controlling RNA trafficking from nucleus to cytoplasm. This is the first report describing a modulation for this protein in asthma. ${ }^{30}$ 
Alpha-enolase was found to be overexpressed in the acute asthma model. This protein is an autoantigen that can give rise to significant titers of antialpha-enolase autoantibody. Interestingly, these antialpha-enolase antibodies have been recognized as being a hallmark of severe asthma. ${ }^{31}$ Our work is the first report suggesting an up-regulation of this protein in acute asthma, and one could speculate that such an overexpression might be triggering autoantibodies production in asthma. However, this hypothesis has to be confirmed, and the precise role of alphaenolase is still to be unveiled.

Apolipoprotein A1 is down-regulated in our chronic asthma model. This finding is in line with a very recent report showing that this protein is also down-regulated in chronic human COPD. ${ }^{32}$ There are indeed many indications in the current literature for a protective role of apolipoprotein A1 against inflammatory diseases such as LPS-induced inflammation. ${ }^{33}$ Moreover, a recent report shows that use of apolipoprotein A1 to remove the pro-inflammatory lipids in a mouse model of asthma provokes an amelioration of asthma phenotype, therefore confirming that studying this protein might be interesting in asthma. ${ }^{34}$

Another chaperone protein, calreticulin is down-regulated in mice chronically exposed to allergen. Calreticulin is mainly located inside the endoplasmic reticulum and interacts with calcium. ${ }^{35}$ This finding is not in line with a previous report from Kypreou et al. showing that calreticulin was up-regulated in a model of bleomycin-induced lung fibrosis ${ }^{36}$ and after cigarettesmoke exposure. ${ }^{37}$ Since membrane-bound calreticulin is a mediator favoring efferocytosis, ${ }^{38}$ one can hypothesize that decreased levels of this protein found in the lungs from allergen-exposed mice might contribute to a perpetuation of the inflammation rendering the phagocytosis of apoptotic cells less efficient. Further studies including a study of the clearance of apoptotic cells are needed to validate this hypothesis.

In conclusion, we report that a $2 \mathrm{D}$-DIGE proteomic study allows the discovery of many potential mediators of asthma with a molecular weight $>20 \mathrm{kDa}$. Among those, we report the increased expression of GRP78 whose inhibition leads to a significant decrease of bronchial hyper-responsiveness and inflammation. This finding validates the use of proteomics to unveil potential new targets in inflammatory diseases and strongly suggests a role for this protein in asthma physiopathology that warrants considering it as a potential new therapeutic target in asthma.

\section{ASSOCIATED CONTENT}

\section{S Supporting Information}

Supplemental figures. This material is available free of charge via the Internet at http://pubs.acs.org.

\section{AUTHOR INFORMATION}

\section{Corresponding Author}

*Didier Cataldo, University of Liege, Tower of Pathology (B23), 4000 Liege, Belgium. Phone: +3243662569 . Fax: +3243662936.

E-mail: didier.cataldo@ulg.ac.be.

\section{Author Contributions}

${ }^{\ddagger}$ The two first and last authors contributed equally to this manuscript.

\section{ACKNOWLEDGMENT}

This work was supported by the Communauté française de Belgique (Actions de Recherches Concertées), the Fonds de la Recherche Scientifique Médicale, the Fonds National de la Recherche Scientifique (F.N.R.S., Belgium), the Fonds spéciaux de la Recherche (University of Liège), the Fondation Léon Fredericq (University of Liège), the DGO6 from the "Région Wallonne" (Belgium), and the interuniversity Attraction Poles Program - Belgian Science Policy IUAP program (Brussels, Belgium). We also thank Sandra Ormenese and the Cell Imaging and Flow Cytometry GIGA Technological Platform for FACS analyses. We thank Fabienne Perin and Christine Fink for their excellent technical assistance.

\section{ABBREVIATIONS}

OVA, ovalbumin; 2D-DIGE, two-dimensional differential in-gel electrophoresis; ACN, acetonitrile; DTT, dithiothreitol; EDTA, ethylenediaminetetraacetic acid; IEF, isoelectric focusing; IPG, immobilized $\mathrm{pH}$ gradient; MALDI-TOF-MS, matrix-assisted laser desorption/ionization-mass spectrometry; PBS, phosphate buffer saline; PMF, peptide mass fingerprint; SDS, sodium dodecyl sulfate; TOF, time-of-flight; IM, interstitial macrophage; AM, alveolar macrophage; STE, short-term exposure; LTE, longterm exposure.

\section{REFERENCES}

(1) (a) Dekkers, B. G.; Maarsingh, H.; Meurs, H.; Gosens, R. Airway structural components drive airway smooth muscle remodeling in asthma. Proc. Am. Thorac. Soc. 2009, 6 (8), 683-92. (b) Gueders, M. M.; Bertholet, P.; Perin, F.; Rocks, N.; Maree, R.; Botta, V.; Louis, R.; Foidart, J. M.; Noel, A.; Evrard, B.; Cataldo, D. D. A novel formulation of inhaled doxycycline reduces allergen-induced inflammation, hyperresponsiveness and remodeling by matrix metalloproteinases and cytokines modulation in a mouse model of asthma. Biochem. Pharmacol. 2008, 75 (2), 514-26. (c) Bourdin, A.; Neveu, D.; Vachier, I.; Paganin, F.; Godard, P.; Chanez, P. Specificity of basement membrane thickening in severe asthma. J. Allergy Clin. Immunol. 2007, 119 (6), 1367-74. (d) Broide, D. H. Immunologic and inflammatory mechanisms that drive asthma progression to remodeling. J. Allergy Clin. Immunol 2008, 121 (3), 560-70; quiz 571-2.

(2) Cataldo, D. D.; Tournoy, K. G.; Vermaelen, K.; Munaut, C.; Foidart, J. M.; Louis, R.; Noel, A.; Pauwels, R. A. Matrix metalloproteinase-9 deficiency impairs cellular infiltration and bronchial hyperresponsiveness during allergen-induced airway inflammation. Am. J. Pathol. 2002, 161 (2), 491-8.

(3) Di Valentin, E.; Crahay, C.; Garbacki, N.; Hennuy, B.; Gueders, M.; Noel, A.; Foidart, J. M.; Grooten, J.; Colige, A.; Piette, J.; Cataldo, D. New asthma biomarkers: lessons from murine models of acute and chronic asthma. Am. J. Physiol. Lung Cell Mol. Physiol. 2009, 296 (2), L185-97.

(4) Gueders, M. M.; Paulissen, G.; Crahay, C.; Quesada-Calvo, F.; Hacha, J.; Van Hove, C.; Tournoy, K.; Louis, R.; Foidart, J. M.; Noel, A.; Cataldo, D. D. Mouse models of asthma: a comparison between C57BL/ 6 and BALB/c strains regarding bronchial responsiveness, inflammation, and cytokine production. Inflamm. Res. 2009, 58 (12), 845-54.

(5) Van Hove, C. L.; Maes, T.; Cataldo, D. D.; Gueders, M. M.; Palmans, E.; Joos, G. F.; Tournoy, K. G. Comparison of acute inflammatory and chronic structural asthma-like responses between C57BL/6 and BALB/c mice. Int. Arch. Allergy Immunol. 2009, 149 (3), 195-207.

(6) Calvo, F. Q.; Fillet, M.; de Seny, D.; Meuwis, M. A.; Maree, R.; Crahay, C.; Paulissen, G.; Rocks, N.; Gueders, M.; Wehenkel, L.; Merville, M. P.; Louis, R.; Foidart, J. M.; Noel, A.; Cataldo, D. Biomarker discovery in asthma-related inflammation and remodeling. Proteomics 2009, 9 (8), 2163-70. 
(7) Jamesdaniel, S.; Salvi, R.; Coling, D. Auditory proteomics: methods, accomplishments and challenges. Brain Res. 2009, 1277, 24-36.

(8) Maes, T.; Provoost, S.; Lanckacker, E. A.; Cataldo, D. D.; Vanoirbeek, J. A.; Nemery, B.; Tournoy, K. G.; Joos, G. F. Mouse models to unravel the role of inhaled pollutants on allergic sensitization and airway inflammation. Respir Res. 2010, 11, 7.

(9) (a) Fajardo, I.; Svensson, L.; Bucht, A.; Pejler, G. Increased levels of hypoxia-sensitive proteins in allergic airway inflammation. Am. L. Respir Crit. Care Med. 2004, 170 (5), 477-84. (b) Fehniger, T. E.; Sato-Folatre, J. G.; Malmstrom, J.; Berglund, M.; Lindberg, C.; Brange, C.; Lindberg, H.; Marko-Varga, G. Exploring the context of the lung proteome within the airway mucosa following allergen challenge. I. Proteome Res. 2004, 3 (2), 307-20. (c) Jeong, H.; Rhim, T.; Ahn, M. H.; Yoon, P. O.; Kim, S. H.; Chung, I. Y.; Uh, S.; Kim, S. I.; Park, C. S. Proteomic analysis of differently expressed proteins in a mouse model for allergic asthma. I. Korean Med. Sci. 2005, 20 (4), 579-85.

(10) (a) Haenen, S.; Vanoirbeek, J. A.; De Vooght, V.; Maes, E.; Schoofs, L.; Nemery, B.; Hoet, P. H.; Clynen, E. Proteome analysis of multiple compartments in a mouse model of chemical-induced asthma. L. Proteome Res. 2010, 9 (11), 5868-76. (b) Houtman, R.; Krijgsveld, J.; Kool, M.; Romijn, E. P.; Redegeld, F. A.; Nijkamp, F. P.; Heck, A. J.; Humphery-Smith, I. Lung proteome alterations in a mouse model for nonallergic asthma. Proteomics 2003, 3 (10), 2008-18.

(11) Wu, C. C.; Chien, K. Y.; Tsang, N. M.; Chang, K. P.; Hao, S. P.; Tsao, C. H.; Chang, Y. S.; Yu, J. S. Cancer cell-secreted proteomes as a basis for searching potential tumor markers: nasopharyngeal carcinoma as a model. Proteomics 2005, 5 (12), 3173-82.

(12) Roh, G. S.; Shin, Y.; Seo, S. W.; Yoon, B. R.; Yeo, S.; Park, S. J.; Cho, J. W.; Kwack, K. Proteome analysis of differential protein expression in allergen-induced asthmatic mice lung after dexamethasone treatment. Proteomics 2004, 4 (11), 3318-27.

(13) Verrills, N. M.; Irwin, J. A.; Yan, He, X.; Wood, L. G.; Powell, H.; Simpson, J. L.; McDonald, V. M.; Sim, A.; Gibson, P. G. Identification of novel diagnostic biomarkers for asthma and chronic obstructive pulmonary disease. Am. J. Respir. Crit. Care Med. 2011, 183 (12), 1633-43.

(14) Petrak, J.; Ivanek, R.; Toman, O.; Cmejla, R.; Cmejlova, J.; Vyoral, D.; Zivny, J.; Vulpe, C. D. Deja vu in proteomics. A hit parade of repeatedly identified differentially expressed proteins. Proteomics 2008, 8 (9), 1744-9.

(15) Hotamisligil, G. S. Endoplasmic reticulum stress and atherosclerosis. Nat. Med. 2010, 16 (4), 396-9.

(16) (a) Imai, Y.; Soda, M.; Inoue, H.; Hattori, N.; Mizuno, Y.; Takahashi, R. An unfolded putative transmembrane polypeptide, which can lead to endoplasmic reticulum stress, is a substrate of Parkin. Cell 2001, 105 (7), 891-902. (b) Nakagawa, T.; Zhu, H.; Morishima, N.; Li, E.; Xu, J.; Yankner, B. A.; Yuan, J. Caspase-12 mediates endoplasmicreticulum-specific apoptosis and cytotoxicity by amyloid-beta. Nature 2000, 403 (6765), 98-103.

(17) (a) Lee, A. S. GRP78 induction in cancer: therapeutic and prognostic implications. Cancer Res. 2007, 67 (8), 3496-9. (b) Li, J.; Lee, A. S. Stress induction of GRP78/BiP and its role in cancer. Curr. Mol. Med. 2006, 6 (1), 45-54.

(18) (a) Gonzalez-Gronow, M.; Selim, M. A.; Papalas, J.; Pizzo, S. V. GRP78: a multifunctional receptor on the cell surface. Antioxid. Redox. Signal. 2009, 11 (9), 2299-306. (b) Philippova, M.; Ivanov, D.; Joshi, M. B.; Kyriakakis, E.; Rupp, K.; Afonyushkin, T.; Bochkov, V.; Erne, P.; Resink, T. J. Identification of proteins associating with glycosylphosphatidylinositol- anchored T-cadherin on the surface of vascular endothelial cells: role for $\mathrm{Grp} 78 / \mathrm{BiP}$ in $\mathrm{T}$-cadherin-dependent cell survival. Mol. Cell. Biol. 2008, 28 (12), 4004-17. (c) Zhang, L. H.; Zhang, X. Roles of GRP78 in physiology and cancer. J. Cell Biochem. 2010, 110 (6), 1299-305. (d) Quinones, Q. J.; de Ridder, G. G.; Pizzo, S. V. GRP78: a chaperone with diverse roles beyond the endoplasmic reticulum. Histol. Histopathol. 2008, 23 (11), 1409-16.

(19) Misra, U. K.; Pizzo, S. V. Ligation of cell surface GRP78 with antibody directed against the COOH-terminal domain of GRP78 suppresses Ras/MAPK and PI 3-kinase/AKT signaling while promoting caspase activation in human prostate cancer cells. Cancer Biol. Ther. 2010, 9 (2), 142-52.

(20) Murphy, D. M.; O'Byrne, P. M. Recent advances in the pathophysiology of asthma. Chest 2010, 137 (6), 1417-26.

(21) (a) Hardy, B.; Battler, A.; Weiss, C.; Kudasi, O.; Raiter, A. Therapeutic angiogenesis of mouse hind limb ischemia by novel peptide activating GRP78 receptor on endothelial cells. Biochem. Pharmacol. 2008, 75 (4), 891-9. (b) Kern, J.; Untergasser, G.; Zenzmaier, C.; Sarg, B.; Gastl, G.; Gunsilius, E.; Steurer, M. GRP-78 secreted by tumor cells blocks the antiangiogenic activity of bortezomib. Blood 2009, 114 (18), 3960-7.

(22) Rosati, E.; Sabatini, R.; Rampino, G.; De Falco, F.; Di Ianni, M.; Falzetti, F.; Fettucciari, K.; Bartoli, A.; Screpanti, I.; Marconi, P. Novel targets for endoplasmic reticulum stress-induced apoptosis in B-CLL. Blood 2010.

(23) Bani, D.; Giannini, L.; Ciampa, A.; Masini, E.; Suzuki, Y.; Menegazzi, M.; Nistri, S.; Suzuki, H. Epigallocatechin-3-gallate reduces allergen-induced asthma-like reaction in sensitized guinea pigs. J. Pharmacol. Exp. Ther. 2006, 317 (3), 1002-11.

(24) Luo, S.; Mao, C.; Lee, B.; Lee, A. S. GRP78/BiP is required for cell proliferation and protecting the inner cell mass from apoptosis during early mouse embryonic development. Mol. Cell. Biol. 2006, 26 (15), 5688-97.

(25) Turano, C.; Coppari, S.; Altieri, F.; Ferraro, A. Proteins of the PDI family: unpredicted non-ER locations and functions. J. Cell Physiol. 2002, 193 (2), 154-63.

(26) (a) Herbon, N.; Werner, M.; Braig, C.; Gohlke, H.; Dutsch, G.; Illig, T.; Altmuller, J.; Hampe, J.; Lantermann, A.; Schreiber, S.; Bonifacio, E.; Ziegler, A.; Schwab, S.; Wildenauer, D.; van den Boom, D.; Braun, A.; Knapp, M.; Reitmeir, P.; Wjst, M. Highresolution SNP scan of chromosome $6 \mathrm{p} 21$ in pooled samples from patients with complex diseases. Genomics 2003, 81 (5), 510-8. (b) Hui, J.; Palmer, L. J.; James, A. L.; Musk, A. W.; Beilby, J. P. AluyMICB dimorphism within the class I region of the major histocompatibility complex is associated with asthma and airflow obstruction in the Busselton population. Clin. Exp. Allergy 2006, 36 (6), 728-34.

(27) Camors, E.; Monceau, V.; Charlemagne, D. Annexins and $\mathrm{Ca}^{2+}$ handling in the heart. Cardiovasc. Res. 2005, 65 (4), 793-802.

(28) Bode, G.; Luken, A.; Kerkhoff, C.; Roth, J.; Ludwig, S.; Nacken, W. Interaction between S100A8/A9 and annexin A6 is involved in the calcium-induced cell surface exposition of S100A8/A9. J. Biol. Chem. 2008, 283 (46), 31776-84.

(29) Halayko, A. J.; Ghavami, S. S100A8/A9: a mediator of severe asthma pathogenesis and morbidity? Can. J. Physiol. Pharmacol. 2009, 87 (10), 743-55.

(30) Ma, A. S.; Moran-Jones, K.; Shan, J.; Munro, T. P.; Snee, M. J.; Hoek, K. S.; Smith, R. Heterogeneous nuclear ribonucleoprotein A3, a novel RNA trafficking response element-binding protein. J. Biol. Chem. 2002, 277 (20), 18010-20.

(31) Nahm, D. H.; Lee, K. H.; Shin, J. Y.; Ye, Y. M.; Kang, Y.; Park, $\mathrm{H}$. S. Identification of alpha-enolase as an autoantigen associated with severe asthma. I. Allergy Clin. Immunol. 2006, 118 (2), 376-81.

(32) Nicholas, B. L.; Skipp, P.; Barton, S.; Singh, D.; Bagmane, D.; Mould, R.; Angco, G.; Ward, J.; Guha-Niyogi, B.; Wilson, S.; Howarth, P.; Davies, D. E.; Rennard, S.; O'Connor, C. D.; Djukanovic, R. Identification of lipocalin and apolipoprotein A1 as biomarkers of chronic obstructive pulmonary disease. Am. J. Respir. Crit. Care Med. 2010, 181 (10), 1049-60.

(33) Li, Y.; Dong, J. B.; Wu, M. P. Human ApoA-I overexpression diminishes LPS-induced systemic inflammation and multiple organ damage in mice. Eur. I. Pharmacol. 2008, 590 (1-3), 417-22.

(34) Nandedkar, S. D.; Weihrauch, D.; Xu, H.; Shi, Y.; Feroah, T.; Hutchins, W.; Rickaby, D. A.; Duzgunes, N.; Hillery, C. A.; Konduri, K. S.; Pritchard, K. A., Jr. D-4F, an apoA-1 mimetic, decreases airway hyperresponsiveness, inflammation, and oxidative stress in a murine model of asthma. I. Lipid Res. 2011, 52 (3), 499-508. 
(35) Coe, H.; Michalak, M. Calcium binding chaperones of the endoplasmic reticulum. Gen. Physiol. Biophys. 2009, 28 (Spec No Focus), F96-F103.

(36) Kypreou, K. P.; Kavvadas, P.; Karamessinis, P.; Peroulis, M.; Alberti, A.; Sideras, P.; Psarras, S.; Capetanaki, Y.; Politis, P. K.; Charonis, A. S. Altered expression of calreticulin during the development of fibrosis. Proteomics 2008, 8 (12), 2407-19.

(37) Kelsen, S. G.; Duan, X.; Ji, R.; Perez, O.; Liu, C.; Merali, S. Cigarette smoke induces an unfolded protein response in the human lung: a proteomic approach. Am. J. Respir. Cell Mol. Biol. 2008, 38 (5), 541-50.

(38) Gardai, S. J.; McPhillips, K. A.; Frasch, S. C.; Janssen, W. J.; Starefeldt, A.; Murphy-Ullrich, J. E.; Bratton, D. L.; Oldenborg, P. A.; Michalak, M.; Henson, P. M. Cell-surface calreticulin initiates clearance of viable or apoptotic cells through trans-activation of LRP on the phagocyte. Cell 2005, 123 (2), 321-34. 